\title{
RESPONSE OF NEUTRON DETECTORS TO HIGH-ENERGY MIXED RADIATION FIELDS
}

\author{
S. Mayer ${ }^{1, *}$, D. Forkel-Wirth ${ }^{2}$, M. Fuerstner ${ }^{2}$, H. G. Menzel ${ }^{2}$, M. J. Mueller ${ }^{2}$, D. Perrin², \\ C. Theis $^{2}$ and H. Vincke ${ }^{2}$ \\ ${ }^{1}$ Paul Scherrer Institut, CH-5232 Villigen PSI, Switzerland \\ ${ }^{2}$ CERN, CH-1211 Geneva 23, Switzerland
}

\begin{abstract}
Radiation protection around CERN's high-energy accelerators represents a major challenge due to the presence of complex, mixed radiation fields. Behind thick shielding neutrons dominate and their energy ranges from fractions of eV to about $1 \mathrm{GeV}$. In this work the response of various portable detectors sensitive to neutrons was studied at CERN's High-Energy Reference Field Facility (CERF). The measurements were carried out with conventional rem counters, which usually cover neutron energies up to $20 \mathrm{MeV}$, the Thermo WENDI-2, which is specified to measure neutrons up to several GeV, and a tissue-equivalent proportional counter. The experimentally determined neutron dose equivalent results were compared with Monte Carlo (MC) simulations. Based on these studies field calibration factors can be determined, which result in a more reliable estimate of $H^{*}(10)$ in an unknown, but presumably similar high-energy field around an accelerator than a calibration factor determined in a radiation field of a reference neutron source.
\end{abstract}

\section{INTRODUCTION}

Radiation protection around CERN's high-energy accelerators presents a major technical and metrological challenge due to the presence of complex, mixed radiation fields. The particle and energy composition of such fields at given points in or outside the accelerator tunnel strongly depend on the measurement position with respect to the beam loss and the kind of shielding used to attenuate the radiation. Behind thick lateral shielding neutrons dominate and their energy ranges from fractions of $\mathrm{eV}$ to several GeV. They are accompanied by photons with energies mainly below $10 \mathrm{MeV}$, by muons and charged hadrons with energies up to several $100 \mathrm{MeV}$.

At CERN various portable neutron counters are in use for operational radiation protection around the high-energy accelerators. The minimum requirements for portable neutron detectors are summarized in the international standard IEC $61005^{(1)}$, which describes portable neutron ambient dose equivalent ratemeters for use in radiation protection. However, the requirements are only defined for neutron radiation of energy up to $16 \mathrm{MeV}$ and which is encountered mainly in nuclear industry. For devices to be used in pulsed fields or fields with higher energies, it is strongly recommended that the user determines the correct operation of the device under the conditions concerned. Consequently, an intercomparison of the response of all CERN neutron detectors was performed at CERN's High-Energy Reference Field Facility $(\mathrm{CERF})^{(2)}$, where the stray radiation field contains neutrons with energies up to $\mathrm{GeV}$.

At CERF, the neutron dose equivalent was measured for different beam intensities at reference

${ }^{*}$ Corresponding author: Sabine.Mayer@psi.ch positions behind the concrete shielding. The instruments used in this study were rem counters like the Thermo WENDI-2, the EG\&G Berthold LB6411, the Studsvik 2202D and the Centronics REM Ionisation Chamber (RIC). With exception of the WENDI-2 which is specified to measure neutrons up to several $\mathrm{GeV}$ all detectors belong to the category of conventional neutron dose rate monitors which usually cover neutron energies up to $20 \mathrm{MeV}$. In addition, measurements were carried out with the HANDI-TEPC (tissue-equivalent proportional counter), which has shown to reliably measure the total dose equivalent in radiation fields with a dominant dose equivalent component due to high-energy particles $^{(3)}$.

\section{INSTRUMENTS}

\section{Berthold LB 6411}

The Berthold neutron probe LB $6411^{(4)}$ is designed to measure the neutron ambient dose equivalent $H^{*}(10)$ within an energy range from thermal energies up to $20 \mathrm{MeV}$. It consists of a cylindrical ${ }^{3} \mathrm{He}$-proportional counter tube surrounded by a polyethylene moderator sphere. In order to measure $H^{*}(10)$ a calibration factor of $0.33 \pm 0.03 \mathrm{nSv} /$ count was applied, which was determined in a field of ${ }^{238} \mathrm{PuBe}$ prior to the measurements.

\section{Studsvik rem counter of type 2202 D}

The Studsvik rem counter of type 2202 D contains $\mathrm{a} \mathrm{BF}_{3}$ proportional counter as thermal neutron counter in the centre of a moderator of polyethylene and boron plastic. The instrument is known to measure $H^{*}(10)$ for neutrons in the energy range from 
thermal to $17 \mathrm{MeV}^{(5)}$. The calibration of the detector in the field of ${ }^{238} \mathrm{PuBe}$ resulted in a calibration factor of $1.54 \pm 0.09 \mathrm{nSv} /$ count.

\section{RIC}

The RIC represents a modified Anderson and Braun rem counter ${ }^{(6)}$, where the inner thermal detector is replaced by a $\mathrm{BF}_{3}$-filled ionisation chamber. Here, the ionisation current produced in the $\mathrm{BF}_{3}$ tube is measured instead of counting pulses from the $(n, \alpha)$ reaction. Hence, the possibility to discriminate against photon-induced signals is lost but the response to pulsed fields increased. The chamber can be used to evaluate the neutron component of the total dose equivalent to an acceptable degree of accuracy in the energy range from thermal to $15 \mathrm{MeV}$. A calibration factor of $106.4 \pm 8.3 \mathrm{nSv} /$ count was determined in the field of a ${ }^{238} \mathrm{PuBe}$ source.

\section{WENDI-2}

The Thermo Wide Energy Neutron Detection Instrument (WENDI-2) ${ }^{(7)}$ consists of a cylindrical polyethylene moderator assembly, which surrounds a ${ }^{3} \mathrm{He}$ counter tube. Inside the moderator the counter tube itself is additionally surrounded by a layer of tungsten powder. This additional tungsten layer has the purpose to generate spallation neutrons, thus enhancing the detector response to neutrons with energies beyond $8 \mathrm{MeV}$. The instrument is designed for measuring the neutron dose equivalent within an energy range from thermal to $5 \mathrm{GeV}$.

\section{HANDI-TEPC}

The HANDI instrument ${ }^{(8)}$, developed by the University of Saarland (Germany), uses a low pressure tissue-equivalent proportional counter (TEPC) and a data processing system based on 16 channel pulsedheight analysis of nearly logarithmically increasing width. The system measures microdosimetric spectra in terms of lineal energy for all components in the radiation field. The neutron component of the dose equivalent can be separately evaluated due to the difference in LET and thus pulse height in the TEPC between neutron secondaries and other radiation components $^{(9)}$.

\section{MEASUREMENTS AND DISCUSSION}

\section{Measurements at the CERF facility}

The response of four rem counters (Berthold LB 6411, Studsvik 2202 D, RIC and WENDI-2) and the HANDI-TEPC was investigated at the CERN-EU high-energy Reference Facility $(\mathrm{CERF})^{(2)}$. CERF enables to generate various types of mixed radiation
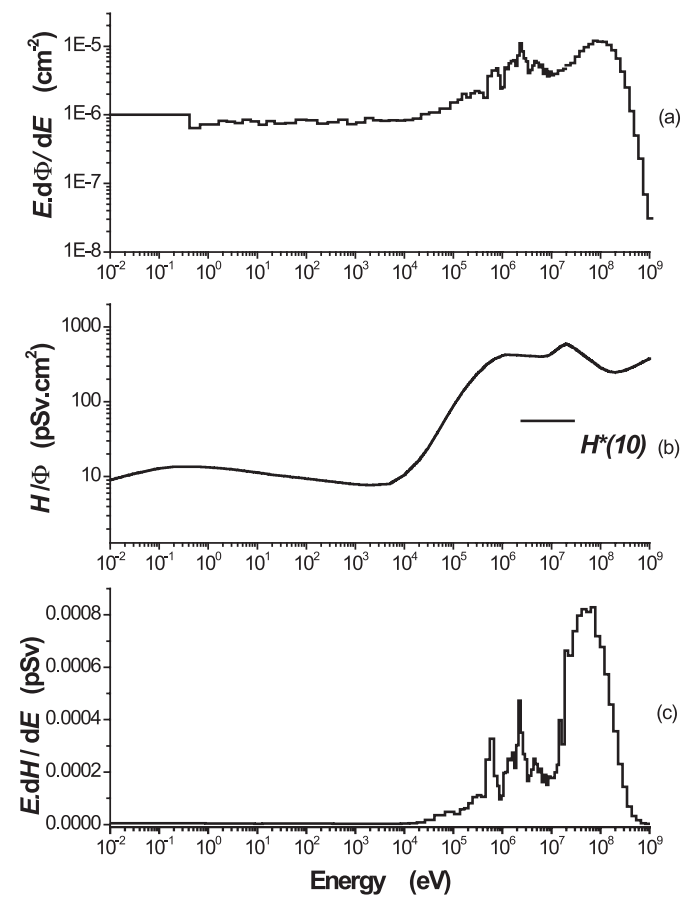

Figure 1. The simulated sneutron fluence spectrum (a) was folded with the conversion function for $H^{*}(10)$ according to Pellicioni ${ }^{(10)}$ (b). Thus, the dose equivalent spectrum is obtained (c).

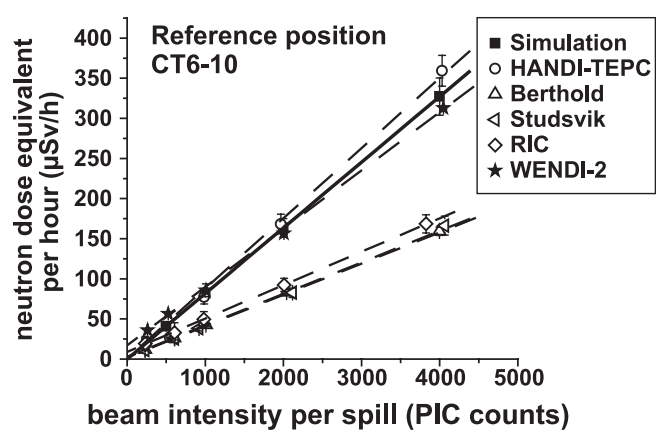

Figure 2. Neutron dose equivalent measured with four types of rem counters and the HANDI-TEPC behind concrete shielding at CERF. For comparison results of recent $\mathrm{MC}$ simulations are shown. One PIC count corresponds to $(2.2 \pm 0.1) \times 10^{4}$ particles impinging on the $\operatorname{target}^{(11)}$.

fields under well controlled conditions by irradiating a copper target with hadrons of high momentum $(120 \mathrm{GeV} / c)$. The mixed fields are composed mainly of neutrons and photons ranging from thermal energies up to $\mathrm{GeV}$. All the instruments were investigated at different beam intensities on top of the $80 \mathrm{~cm}$ 
Table 1. Neutron dose equivalent per hour evaluated for a beam intensity of 1000 PIC counts per spill at the reference position CT6-10 at CERF. The indicated values are averaged over the intensities after background subtraction. The relative deviations between the results of the instruments and MC simulations of 2004 are calculated. The last column indicates the calibration source and the calibration factor of the rem counters.

\begin{tabular}{lccc} 
Instrument & $\begin{array}{c}\text { Neutron dose equivalent } \\
\text { per hour }(\mu \mathrm{Sv} / \mathrm{h})\end{array}$ & $\begin{array}{c}\text { Relative deviation between instrument } \\
\text { and simulation 2004 }(\%)\end{array}$ & $\begin{array}{c}\text { Calibration source } \\
\text { and factor (nSv/count) }\end{array}$ \\
\hline Simulation 2004 & $84.8 \pm 3.0$ & - & 2.9 \\
HANDI-TEPC & $87.3 \pm 7.5$ & -53.9 & $238 \mathrm{PuBe} 0.33 \pm 0.03$ \\
Berthold LB6411 & $39.1 \pm 0.5$ & -54.6 & $238 \mathrm{PuBe} 1.54 \pm 0.09$ \\
Studsvik 2202D & $38.5 \pm 0.6$ & -40.8 & $238 \mathrm{PuBe} 106.4 \pm 8.3$ \\
RIC & $50.2 \pm 4.0$ & -11.9 & $252 \mathrm{Cf} 0.32 \pm 0.01$ \\
WENDI-2 & $74.7 \pm 4.6$ & & \\
\hline
\end{tabular}

thick concrete shielding at the reference position CT 6-10 (Concrete Top 6-10). In Figure 1, the simulated neutron fluence spectrum together with the $H^{*}(10)$ conversion function over energy ${ }^{(10)}$ and the resulting dose equivalent spectrum are shown. From this figure one can see that the main contribution to the total dose equivalent is produced by neutrons with energies higher than $20 \mathrm{MeV}$. In Figure 2 and Table 1 the neutron dose equivalent per hour obtained at CT6-10 are presented. All results are normalised to the CERF beam monitor, an air-filled precision ionisation chamber (PIC). One PIC count corresponds to $(2.2 \pm 0.1) \times 10^{4}$ particles impinging on the target $^{(11)}$. In addition to the measurement results, the neutron dose equivalent values were estimated by recent Monte Carlo (MC) simulations using the latest version of the FLUKA code ${ }^{(12,13)}$.

\section{CONCLUSION}

Four different types of rem counters and the HANDI-TEPC were compared in the CERF-field behind the concrete shielding at different beam intensities. The HANDI-TEPC and the WENDI-2 agree with the neutron dose equivalent obtained by MC simulations within $\pm 12 \%$. The international standard IEC 61005 recommends a deviation to the actual neutron dose equivalent of less than $\pm 30 \%$ as acceptable for neutron energies up to $16 \mathrm{MeV}$. The study at the CERF facility showed that the WENDI-2 complies with the international standard IEC 61005 even in mixed radiation fields with particle energies up to a few GeV. As expected, the conventional types of rem counters, which were designed for neutron energies up to $20 \mathrm{MeV}$ and which are calibrated in radiation fields of reference sources, underestimate the neutron dose equivalent by a factor of two. Nevertheless, since it is proven that FLUKA is capable to simulate correctly the radiation field at the concrete top positions ${ }^{(14)}$ a field calibration factor for each detector can be deduced. This field calibration factor provides a better estimate of
$H^{*}(10)$ in a similar, but unknown high-energy field around an accelerator than a calibration factor determined in a radiation field of a reference neutron source.

\section{REFERENCES}

1. International Standard, IEC 61005. Portable neutron ambient dose equivalent ratemeters for use in radiation protection (1990).

2. Mitaroff, A. and Silari, M. The CERN-EU high-energy reference field (CERF) facility for dosimetry at commercial flight altitudes and in space. Radiat. Prot. Dosim. 102, 7-22 (2002).

3. Schrewe, U. J., Alberts, W. G., Alvera, A. V., Ferrari, A., Otto, T. and Silari, M. Calibration problems, calibration procedures and reference fields for dosimetry at flight altitudes. Radiat. Prot. Dosim. 86, 289-295 (1999).

4. Klett, A. and Burgkhardt, B. The new remcounter LB6411: measurement of neutron ambient dose equivalent $H^{*}(10)$ according to ICRP60 with high sensitivity. IEEE Trans. Nucl. Sci. 44, 757-759 (1997).

5. Studsvik. Manual of the Neutron Dose Rate Meter 2202 D. (Sweden) (1973).

6. Andersson, I. O. and Braun, J. A. Neutron rem counter with uniform sensitivity form $0.025 \mathrm{eV}$ to $10 \mathrm{MeV}$. In: Proceedings of the IAEA Symposium on Neutron Dosimetry. (Vienna) pp. 87-95 (1963).

7. Olsher, R. H., Hsu, H.-H., Beverding, A., Kleck, J. H., Casson, W. H., Vasilik, D. G. and Devine R. T. WENDI: an improved neutron rem meter. Health Phys. 79, 170-181 (2000).

8. Kunz, A., Arend, E., Dietz, E., Gerdung, S., Grillmaier, R. E., Lim, T. and Pihet, P. The homburg area neutron dosemeter HANDI: characteristics and optimisation of the operational instrument. Radiat. Prot. Dosim. 44, 213-218 (1992).

9. Schmitz, Th., Waker, A. J., Kliauga, P. and Zoetelief, Eds. EURADOS Report. Design, Construction and use of Tissue Equivalent Proportional Counters. Radiat. Prot. Dosim. 61 (1995).

10. Pelliccioni, M. Overview of fluence-to-effective dose and fluence-to-ambient dose equivalent conversion coefficients for high energy radiation calculated using the FLUKA code. Radiat. Prot. Dosim. 88, 279-297 (2000). 


\section{S. MAYER ET AL}

11. Vincke, H., Mayer, S., Efthymiopoulos, I., Fabich, A., Forkel-Wirth, D., Müller, M. J. and Theis, C. Accurate PIC calibration by the use of a coincidence of two scintillators. Technical Note, CERN-SC-2004-90RP-TN (2004).

12. Fassò, A., Ferrari, A. and Sala, P. R. Electron-photon transport in FLUKA. status. In: Proceedings of the Monte Carlo 2000 Conference, Lisbon, October 23-26 2000. Kling, A., Barao, F., Nakagawa, M., Tavora, L. and Vaz, P. Eds. (Berlin: Springer-Verlag), pp. 159-164 (2001).
13. Fassò, A., Ferrari Ranft, A. J. and Sala, P. R. FLUKA. status and prospective for hadronic applications. In: Proceedings of the Monte Carlo 2000 Conference, Lisbon, October 23-26 2000. Kling, A., Barao, F., Nakagawa, M., Tavora, L. and Vaz, P. Eds. (Berlin: Springer-Verlag), 955-960 (2001).

14. Theis, C., Forkel-Wirth, D., Perrin, D., Roesler R. and Vincke H. Characterisation of ionisation chambers for a mixed radiation field and investigation of their suitability as radiation monitors for the LHC. Radiat. Prot. Dosim. 116, 170-174 (2005). 